\title{
Unión Europea, administraciones públicas y mercado interno
}

\section{Francesc Morata *}

\section{Introducción}

Al igual que en otros momentos de su corta historia, la Comunidad Europea atraviesa un período de crisis. El debate posterior a Maastricht ha reavivado el tradicional enfrentamiento entre partidarios y adversarios del proceso de integración. A pesar de las recientes reformas institucionales, el talón de Aquiles de la Comunidad sigue siendo el déficit democrático. Por ello, según la expresión acunaada por el anterior Presidente de la Comisión, Jacques Delors, ésta constituye todavía "un objeto político no identificado", un sistema híbrido que oscila entre la confederación y la federación (WALLACE, 1983; SCHMITTER, 1991).

Si bien la "Europa de los Pueblos" parece todavía un objetivo remoto, otra Europa se ha ido haciendo cada vez más tangible: la "Europa de las Administraciones" (SIEDENTOPF y Ziluer, 1988), cuyo funcionamiento afecta diariamente de modo directo al conjunto de los ciudadanos comunitarios y que comprende, no sólo el corazón del sistema administrativo comunitario -la Comisión - sino también la práctica totalidad de las administraciones de los Estados miembros. Por ello, puede decirse que la legitimidad "funcional" actúa hoy por hoy como sustitutivo de la legitimidad democrática (ToONEn, 1992).

El papel determinante de las burocracias en la elaboración de las políticas comunitarias constituye, en efecto, una de las características más acusadas del desarrollo institucional de la Comunidad y resulta indirectamente del déficit democrático: la transferencia de competencias internas a la Comunidad sólo ha sido posible en la medida en que los gobiernos de los Estados miembros tenían garantizado un amplio control sobre las decisiones (WELLER, 1984). La estrecha implicación de las administraciones nacionales en las distintos ámbitos de actividad comunitarios genera un sistema burocrático entrelazado (pag, 1988), una forma particular de federalismo cooperativo (WESSELS, 1985).

Los últimos años han representado un período de innovación política para la Comunidad Europea, materializada por la puesta en práctica de políticas, instituciones y procedimientos en ámbitos tan variados como la política industrial, la tecnología, la educación, la política social, el medio ambiente o la política monetaria. Todo ello refleja un aumento sustancial de las competencias comunitarias. Sin embargo, el problema consiste en saber si existe la capacidad suficiente para ejercerlas de forma efectiva. Para responder a esta pregunta, examinaremos, en primer lugar, la estructura organizativa y las pautas de funcionamiento de la Comisión con objeto de detectar sus principales flaquezas. A continuación, analizaremos el modelo de gestión de las políticas comunitarias, poniendo de relieve algunos de los obstaculos administrativos que dificultan el funcionamiento efectivo del mercado interno. Por último, nos referiremos a algunas iniciativas de la Comisión tendentes a facilitar la implementación de dicha política.

\section{La Comisión: núcleo central de la administración comunitaria}

\section{Caracteristicas principales}

La Comisión no es el gobierno de la Comunidad, pero tampoco es una simple correa de transmisión de la voluntad expresada por los Estados miembros. Por supuesto, la Comisión sufre la mayor parte de los vicios úpicos de cualquier administración pública (rigideces organizativas, coordinación deficiente, rivalidades burocráticas, etc.). Concebida según el modelo de las agencias de planificación francesas de los años cincuenta, la administración comunitaria ha tenido que afrontar en pocos años el doble reto de la extensión de las competencias supranacionales y la entrada de nuevos Estado miembros. Todo ello ha comportado la necesidad de proceder a constantes reajustes, preservando los equilibrios políticos internos (cuotas nacionales), en un contexto caracterizado por la influencia creciente de las administraciones nacionales, único modo de garantizar una coordinación a nivel europeo.

Tildada por los anticomunitarios de administración centralista y depredadora de recursos, de organización hiperreguladora empeñada en mermar la soberanía de los Estados miembros, la Comisión no se corresponde en nada con esta imagen terrorífca (DEwost, 1992).Ciertamente se trata de una organización tec- 
nocrática desprovista de legitimación política directa (MAJONE, 1992). Sin embargo, su existencia resulta fundamental para el proceso de integración europea puesto que ostenta el monopolio de las iniciativas legislativas mediante las proposiciones de reglamento o de directiva dirigidas al Consejo de Ministros. Ejerciendo a fondo dicha prerrogativa, la Comisión ha conseguido introducir a menudo temas fundamentales en la agenda política comunitaria. Por ejemplo, resultaría imposible entender el período de eurodinamismo que se inicia con el Libro Blanco, relativo a la puesta en marcha del Mercado Interno, y el Acta Unica Europea (AUE) y que concluye con la cumbre de Maastricht sin tener en cuenta el papel determinante desempeñado por la Comisión. Esta ha demostrado, en efecto, su capacidad de actuar como un "intermediario honesto" (bonest broker) (DehousSe y Majone, 1993:17), definiendo un interés común -el mercado interno - sin poner en peligro los intereses "vitales" de los Estados miembros. En segundo lugar, la Comisión actúa como guardián de los Trałados, con sus propios medios y en combinación con el Tribunal de Justicia. En tercer lugar, gestiona el presupuesto y la hacienda comunitaria. También negocia y concluye acuerdos internacionales en representación de la Comunidad. Por último, la Comisión es responsable de la implementación de las políticas en los términos que establece el Consejo de Ministros.

Sin embargo, para ejercer dichas funciones, la Comisión depende en gran medida de los Estados miembros. Las iniciativas legislativas resultan de un complejo proceso de consultas con los funcionarios de los distintos países que actúan en calidad de técnicos y expertos. Una vez formulado el borrador de proposición, los comentarios y reservas expresados por los expertos nacionales son objeto de negociaciones mediante procedimientos formales e informales. Este mecanismo que faculta la intervención de las administraciones nacionales se reproduce a gran escala cuando la proposición llega a manos del Comité de Representantes Permanentes de los Estados miembros (COREPER), antes de su aprobación definitiva por el Consejo de Ministros. Como veremos más adelante, la situación de dependencia de la Comisión se acentúa todavía más cuando se trata de poner en práctica las decisiones comunitarias: salvo algunas excepciones, entre las que destacan la política agrícola y el control de libre competencia, la aplicación legal de las normas comunitarias y su implementación efectiva se hallan atribuidas a las administraciones y a los tribunales internos. Por otra parte, sin la información que le suministran tanto las administraciones nacionales como las empresas o los particulares, el control y seguimiento de la normativa comunitaria a cargo de la Comisión resultarían meramente simbólicos. Además, ésta se halla supeditada a la voluntad de los Estados miembros en lo relativo a sus necesidades de financiación. Sin duda, este conjunto de factores impiden hablar de la existencia de un ejecutivo comunitario en sentido estricto (CASSESE, 1988).
La Comisión sufre una serie de condicionamientos cuantitativos y cualitativos que limitan su capacidad de gestión. De hecho, se trata de una organización de dimensiones más bien modestas si nos atenemos a los estándares gubernamentales. Al margen de cuatro mil traductores e intérpretes, el personal de la Comisión suma unos once mil funcionarios, de los que unos cuatro mil ejercen funciones ejecutivas. A pesar del doble impacto de las nuevas políticas incorporadas a la agenda comunitaria y de las sucesivas ampliaciones, la relación existente entre el número de funcionarios y el número de habitantes de la Comunidad no ha variado de forma significativa desde su creación. Ello explica que los efectivos de la Comisión sean comparables a los del Ministerio francés de Cultura e incluso inferiores a los de cualquier capital o región europea (HAY, 1989). Por tanto, no parece descabellado deducir de estas cifras la existencia de un déficit de gestión en el seno de la Comisión.

Sin embargo, la institución se ve afectada también por otros problemas de indole política y organizativa. En primer lugar, cabe recordar el carácter políticamente anómalo de la Comisión $\mathrm{o}$, si se prefiere, su debilidad institucional. Debido al famoso "déficit democrático", la elección del ejecutivo comunitario, entendiendo por éste el Presidente y el Colegio de Comisarios, no es la culminación de un proceso democrático en el que el Parlamento Europeo llevaría la voz cantante, sino de una negociación compleja entre los distintos Estados miembros en la que se trata ante todo de preservar delicados equilibrios, tanto nacionales como ideológicos. Inicialmente, la Comisión fue concebida como un organismo relativamente pequeño dirigido por el Colegio de Comisarios, responsables de definir un marco organizativo eficaz y coherente de cara a la selección de las propuestas políticas prioritarias. Según el Tratado CEE (art. 158), los Comisarios son nombrados de común acuerdo por los Estados miembros. De hecho, no obstante, su designación se efectúa de forma individual por parte de cada gobierno. Por tanto, resulta poco menos que inimaginable suponer una desvinculación de los Comisarios de las respectivas lealtades nacionales.

Ciertamente, el Tratado de Maastricht introduce algunos retoques democratizantes cuando prevé la consulta del Parlamento Europeo previa a la designación del presidente y los Comisarios y el voto de aprobación del propio Parlamento. A pesar de su necesaria independencia formal, no cabe duda de que los Comisarios y, con ellos, los altos funcionarios representan las distintas sensibilidades nacionales, hallándose sujetos incluso a las presiones de sus respectivos gobiernos. Sin embargo, también es cierto que la coexistencia de distintas percepciones en el seno del órgano supranacional suele resultar beneficiosa para el conjunto de los intereses representados puesto que, en cierto modo, actúa como un mecanismo de autocontrol (NUGENT, 1992).

El mantenimiento de las conexiones estatales explica también que la distribución de las carteras entre los Comisarios 
constituya siempre un dificil ejercicio de negociación y de búsqueda de equilibrios políticos entre los gobiernos y entre los propios Comisarios. El Presidente ejerce sin lugar a dudas una influencia significativa; todavía más si dispone de una fuerte personalidad. Sin embargo, sus potestades son limitadas ya que actúa más como un primus inter pares que como un verdadero Presidente de gobierno. Con un mandato de dos años, inferior al de los demás Comisarios (cuatro años), no cuenta con la fuerza política necesaria para formar un equipo acorde con sus preferencias. Si bien desempeña un papel destacado de representación externa y de liderazgo interno, una vez constituido el Colegio de Comisarios, el Presidente no está en condiciones de supervisar y coordinar eficazmente el conjunto de las carteras.

Esta problemática interna se ve considerablemente agravada por la falta de control externo de los distintos Comisarios, por lo que el déficit democrático de la Comunidad repercute en el déficit de gestión (METCALFE, 1992). En efecto, a pesar de la asignación individual de las carteras, los Comisarios son irresponsables individualmente ante el Parlamento Europeo ya que pertenecen a un órgano colegial. Si la Cámara decidiera reprobar la actuación de un Comisario, debería recurrir a un voto de censura contra el conjunto de la Comisión, abriendo así una crisis política de consecuencias imprevisibles. De este modo, la colegialidad se convierte en un obstáculo añadido al control democrático de la Comunidad.

En el plano interno, la Comisión se organiza de forma similar a los gobiernos de los Estados miembros. Sin embargo, se produce una falta de correspondencia númerica entre los veinte Comisarios y las veintitrés Direcciones Generales, las unidades administrativas básicas. La mayor parte de los Comisarios desempeñan varias carteras, lo cual les obliga a relacionarse simultáneamente con varias Direcciones Generales. Por ende, la división de funciones entre las Direcciones Generales y los demás servicios resulta a menudo incoherente. Algunos ejemplos permiten ilustrar dicha afirmación. Si bien existe una DG responsable de la política de medio ambiente (DG XI), los temas medioambientales se reparten de hecho entre diez Direcciones Generales, dos Institutos y una Agencia especializada; las materias relacionadas con la alimentación y el consumo dependen de tres Direcciones Generales; los temas audiovisuales también de tres; y las competencias relativas al tratado CECA, de ocho. El problema adquiere mayor complejidad debido al poder que ejercen los directores generales sobre sus respectivas administraciones y a las reticencias de éstos a cooperar con sus homólogos, incluso cuando se producen solapamientos evidentes. Por ello, y a pesar de sus reducidas dimensiones, la coordinación constituye una preocupación constante para la Comisión. A tal efecto y a lo largo de los años, se han ido estableciendo distintos órganos y procedimientos (en particular, la presidencia, el Colegio de Comisarios, los gabinetes de éstos, la Secretaría General y los numerosos comités ad boc y las llama- das task forces). No obstante, la compartimentación burocrática sigue siendo la característica dominante (NUGENT, 1992), por lo que las conclusiones del Informe Spierenburg de 1979, relativo a la reforma de la institución, todavía siguen siendo vigentes: "(...) lo ciento es que la Comisión se gestiona de un modo y con unas técnicas inapropiados para las actuales circunstancias y la situación puede agravarse todavía más después de una futura ampliación" (SPIERENBURG, 1979).

A lo anterior, cabe añadir una falta de flexibilidad en la politica de personal y de gestión de recursos humanos con objeto de situar a las personas indicadas en los puestos de responsabilidad según las necesidades de cada momento. Por motivos históricos, la movilidad del personal es escasa, no sólo entre Direcciones Generales, sino incluso en el seno de éstas. Las administraciones nacionales no están en modo alguno dispuestas a renunciar a sus cuotas de poder, es decir, a la ocupación de los puestos de dirección estratégicos que les corresponden en virtud de un acuerdo tácito (KASSIM y WRIGHT, 1992). De hecho, el peso cuantitativo y cualitativo del personal comunitario es muy variable, existindo incluso una escala de reputaciones de las distintas DD.GG. que sirve de punto de referencia para los propios Estados miembros a la hora de relacionarse con la Comisión (LODGE, 1993). Ello supone diferencias importantes en cuanto a la calidad de la labor preparatoria de las politicas y a la eficiencia organizativa.

Por último, y debido a factores estructurales, siempre ha existido un desequilibrio importante en la Comisión entre formulación y ejecución de políticas. Ello obedece a la configuración institucional de la Comisión como constante impulsora de nuevas políticas al margen de las implicaciones relativas a su puesta en práctica y gestión efectiva. En otras palabras, la Comisión es ante todo una administración especializada en la elaboración de propuestas políticas (LuDLow, 1992), a pesar de tener encomendadas tareas tan fundamentales como la supervisión y el control de las actividad de transposición y cumplimiento efectivo de las obligaciones comunitarias, para lo cual dispone de medios humanos y materiales claramente insuficientes. Esta sorprendentente inhibición con respecto a la implementación de las políticas responde también a la voluntad de preservación de las potestades sancionadoras propias de la Comisión, con lo cual se produce un "conflicto implícito entre la funciones de gestión y de control" (MetCal.Fe, 1992:126). Sin embargo, como se ha dicho anteriormente, la Comisión es sólo el núcleo central de un sistema de interdependencias en el que las burocracias nacionales juegan igualmente un papel crucial.

\section{La interdependencia administrativa}

La participación de las administraciones nacionales en la gestión de las políticas comunitarias constituye un claro reflejo de la especificidad de la Comunidad Europea y, en especial, de la tensión permanente entre soberanía e integración. Dicha tensión 
permite definir el proceso político comunitario como la gestión de la interdependencia (WALACE 1983). Desde esta perspectiva, a medida que se han ido ampliando sus objetivos e incrementando sus competencias, la necesidad de cooperación con las administraciones y los demás actores nacionales se ha convertido en algo fundamental para la Comunidad (KASSIM y WRIGHT, 1992). Esta tendencia se reproduce prácticamente en todos los estadios del proceso político. En primer lugar, y contrariamente a lo previsto en el Tratado CEE, la Comisión no ejerce un monopolio absoluto con respecto a la iniciación de las políticas. Esta tarea, la lleva a cabo rodeada de una compleja red de comités de consulta compuestos por expertos nacionales, académicos y representantes de intereses públicos y privados, entre los que se incluyen tanto las regiones como las ciudades y sus asociaciones respectivas, organizaciones no gubernamentales y grupos económicos y profesionales. La mayor parte de estos comités constituyen verdaderas comunidades establecidas alrededor de políticas específicas (policy communities) ya que tienen un carácter estable, se reúnen periódicamente y los miembros que los componen son casi siempre los mismos (NUGENT, 1992). Otros se crean ad boc, con objeto de discutir los primeros borradores de proposición. Al margen de estos dos tipos esenciales de comités, aparecen otros de carácter más híbrido con formas de participación mixtas. Así se estima en más de quinientos el número de grupos de expertos establecidos por la Comisión con el doble objetivo de recabar información y anticipar las reacciones de los Estados miembros ante sus propuestas (Wessels, 1990). Como es lógico, los funcionarios nacionales tratan de influir en la Comisión con el objetivo de que la legislación comunitaria incorpore sus puntos de vista; sin embargo, en este estadio, la Comisión actúa autónomamente, tratando de mejorar en lo posible la calidad de los textos, sin alterar las condiciones básicas del consenso. Tras la presentación formal de la proposición al Consejo de Ministros, el máximo protagonismo negociador corresponde a los representantes nacionales. El COREPER (Comité de Representantes Permanentes de los Estados miembros), dividido en dos secciones, I y II, representa la columna vertebral de un intenso proceso de intercambio político en el que participan alrededor de doscientos grupos de trabajo responsables de "digerir" las proposiciones de la Comisión antes de su incorporación a la agenda del Consejo de Ministros. El COREPER I trata los temas de contenido más técnico, incluyendo el presupuesto, mientras que el COREPER II se encarga de los temas políticos e institucionales (GONZÁlEZ, 1992). Hay que tener en cuenta igualmente los contactos que los expertos nacionales mantienen con los miembros del Parlamento Europeo, en su condición de institución participante en el proceso decisorio a través de la emisión de dictámenes o resoluciones, del procedimiento de cooperación o del de codecisión. Así, la intervención formal e informal de los funcionarios expertos de las administraciones nacionales en cada una de las fases de elaboración de las decisiones facilita la formación del consenso interinstitucional hasta los mínimos detalles.

Por otro lado, de acuerdo con lo establecido en los Tratados o por delegación del Consejo de Ministros, la Comisión dispone de potestades reglamentarias materializadas en la emanación de cuatro a cinco mil actos normativos por año. Sin embargo, una vez más, dicha actividad supone la movilización de un ingente número de comités regulativos y de gestión integrados por funcionarios nacionales y presididos por representantes de la Comisión. La capacidad de influencia de ambos es muy parecida: los comités de gestión pueden bloquear las decisiones de la Comisión por mayoría cualificada, mientras que los regulativos deben ratificarlas por igual mayoría. Además, para cada Fondo estructural se han instituido sendos comités de consulta y de gestión, en los que participan representantes de la Comisión y de las autoridades centrales, regionales e incluso, a veces, locales.

La proliferación de este tipo de órganos ha dado lugar a lo que en lenguaje comunitario se denomina el sistema de la "comitología", considerado por muchos autores como una forma de control de la Comisión por los Estados miembros, resultado, a la vez, del déficit de gestión y del déficit democrático (Peters, 1992; Blumann, 1992). No hay duda de que los comités facilitan la aportación de los conocimientos técnicos disponibles en cada administración contribuyendo, al mismo tiempo, a la elaboración del consenso comunitario. También constituyen un medio importante de expresión de la variedad de culturas administrativas y de las diversidades económicas existentes en la Comunidad. No obstante, lo cierto es que, al margen de su eficiencia relativa, la multiplicación de los comités, el solapamiento de sus actividades y la disparidad de las reglas que guían su funcionamiento generan una falta real de transparencia. En estas condiciones, "resulta difícil que los ciudadanos comunitarios consigan identificar el órgano responsable de las decisiones que les afectan y los recursos legales disponibles" (DEHOUSSE y MAJONE, 1992:24).

\section{Los grupos de interés}

Otra de las críticas dirigidas a la Comisión se refiere a su alto grado de permeabilidad respecto a las presiones de los grupos organizados o lobbies (MAZEY y RICHARDSON, 1993). Ello no debe extrañar si tenemos en cuenta las especificidades del sistema comunitario. Por un lado, éste ofrece incentivos suficientes (políticas regulativas, distributivas y redistributivas) para que los grupos organizados traten de incidir en el proceso de decisión y, en particular, en la Comisión en su calidad de elaboradora de políticas complejas en un ambiente altamente competitivo (administraciones públicas, grandes empresas, lobbistas profesionales, etc.). Ello no tendría mayores consecuencias si no fuera porque la escasa legitimidad democrática de la Comunidad obliga a la Comisión a legitimarse constantemente por otras vías: consultando y dando respuesta a las demandas expresadas 
por los grupos más hábiles e influyentes. En otras palabras, la Comisión se ve obligada a satisfacer intereses públicos y privados a escala europea sin disponer de la representatividad propia de los gobiernos nacionales.

La participación de los grupos de interés acentúa todavía más la ya de por sí compleja maquinaria administrativa que sustenta el sistema comunitario. Esta no se limita a los procedimientos formales de los comités consultivos, sino que se extiende a las distintas fases del proceso político. En su condición de lobbies, los grupos de interés están permanentemente dispuestos a establecer contactos formales e informales con la Comisión y los representantes nacionales. De hecho, la Comisión depende en gran medida de la información y de las opiniones del sector privado en la medida en que, como sabemos, no dispone por si sola de la expertise necesaria. De acuerdo con el enfoque neofuncionalista, en su calidad de institución supranacional, incluso tiende a favorecer el lobbismo con el objetivo de vencer las resistencias de los Estados miembros (PAG, 1988).

Los motivos para ejercer una acción de lobbying a nivel comunitario son innumerables ya que están en juego ganancias o pérdidas evidentes en términos de legislación, financiación o influencia política (Van SCHENDElen, 1993). La complejidad misma del sistema decisorio - más fragmentado organizativamente, menos integrado jerárquicamente y más competitivo que los sistemas nacionales - ofrece una accesibilidad mucho mayor (SCHMiTTER y STREEK, 1989). La aprobación del Acta Unica Europea, en 1986, representó un incremento espectacular del número de grupos actuantes en Bruselas. De acuerdo con una Comunicación reciente de la Comisión (Comisión, 1993), se estima en más de tres mil el número de grupos de interés establecidos en Bruselas y en unas diez mil el total de personas dedicadas a esta actividad. Destacan las más de quinientas federaciones internacionales y europeas presentes en representación de unas cinco mil organizaciones nacionales, mientras que doscientas firmas se hallan directamente representadas, además de unos cien despachos profesionales especializados en la gestión de asuntos comunitarios. Estas cifras revelan la importancia creciente que los intereses organizados atribuyen a las decisiones comunitarias, en especial con la puesta en marcha del Mercado Unico.

A pesar de que, en principio, la Comisión no concede privilegios especiales a los grupos, el grado de proliferación alcanzado, la ausencia de reglas de comportamiento específicas, la disparidad de recursos organizativos entre las organizaciones con y sin ánimo de lucro y la complejidad técnica de la mayor parte de las regulaciones comunitarias tienden, sin lugar a dudas, a favorecer a los intereses económicos en detrimento de la transparencia democrática.

Uno de los aspectos más destacados del lobbying comunitario reside en el protagonismo creciente de las instituciones regio- nales y locales. Hasta la fecha, y a la espera de lo que pueda dar de sí el recién constituido Comité de las Regiones, las autoridades regionales se han visto excluidas del proceso de decisión, a pesar de los procedimientos de coordinación establecidos en algunos Estados miembros con el objetivo de definir las posiciones nacionales en temas de su competencia. Ello contrasta fuertemente con el papel de las regiones como implementadores últimos de políticas y programas comunitarios, especialmente en Alemania, España, Bélgica e Italia. Como consecuencia de ello, los gobiemos regionales, que disponen ya de más de cincuenta oficinas de representación en la capital comunitaria, tratan de fortalecer su papel estratégico canalizando las demandas de los intereses territoriales y estableciendo acuerdos de cooperación con sus homólogos en los demás Estados miembros para conseguir una mayor infuencia política y aprovechar las oportunidades de financiación adicional que ofrece la CE (MOUns y MORATA, 1993). La cooperación transregional va incluso más lejos, ya que está generando nuevas formas de gestión basadas en redes interorganizativas con la participación de actores públicos y privados (administraciones, universidades, agentes sociales, asociaciones de intereses y empresas). Dicha tendencia suprarregionalista se refleja, por ejemplo, en los "Cuatro Motores para Europa", un acuerdo de cooperación mutua suscrito en 1988 por los presidentes de Baden-Württemberg, Cataluña, Lombardía y RhôneAlpes, y que abarca ámbitos tan variados como las relaciones económicas exteriores, la cooperación económica, el intercambio de información, la formación de funcionarios, la transferencia de tecnología y el medio ambiente (MORATA, 1994). Las principales ciudades europeas también participan de este nuevo dinamismo, como lo demuestra la red de "Eurociudades", un activo lobby local, ya presente en Bruselas, que agrupa más de cincuenta municipios, con dos objetivos complementarios: articular el espacio comunitario mediante programas de cooperación específicos e inserir los intereses urbanos en las políticas comunitarias (EUROCITIES, 1994).

\section{La implementación de las políticas comunitarias}

\section{Una tarea compleja}

La administración europea abarca al conjunto de los Estados miembros. Ello significa que, en gran medida, las diferencias de rendimiento observables entre las distintas políticas comunitarias obedecen a factores exógenos a la Comisión. A pesar de ello, y de forma sorprendente, el número de estudios empíricos relativos a la implementación de las políticas comunitarias desde una perspectiva comparada sigue siendo muy escaso (Clavarini AZZi, 1986; SIedentopf y Ziller, 1988). 
El análisis de las políticas públicas demuestra que el proceso de ejecución consiste en algo más que en la mera aplicación mecánica de una norma o de un programa, ya que los responsables directos e, incluso, el público interesado disponen de los recursos necesarios para alterar el contenido mismo de la decisión en función de sus valores e intereses y de los condicionamientos que pesan sobre su actuación (MÉNY y THOENIG, 1992). Esta situación aparece todavía más evidente en el espacio comunitario por razón de la gran variedad de sistemas políicos, culturas administrativas y actores implicados en los distintos niveles de gobierno. Si bien los mecanismos de implementación de las políticas comunitarias se asemejan en gran medida a los de un sistema federal (ejecución y gestión totalmente descentralizadas con arreglo al marco normativo común establecido por las directivas y control jurisdiccional a cargo del Tribunal de Justicia), la Comisión no dispone de los medios necesarios para garantizar una aplicación efectiva y uniforme. Como hemos visto, se trata de una organización excesivamente reducida, desprovista de los medios de gestión y de coordinación imprescindibles para asumir un control jerárquico de tipo tradicional. Por ejemplo, no puede remitir instrucciones a los órganos ejecutivos de los Estados miembros ni tampoco asignarles tareas específicas. Por ello, las funciones de control y seguimiento de la Comisión son de una enorme complejidad (Comisión, 1993a). En la medida de lo posible, ésta analiza la pertinencia de las medidas de ejecución adoptadas por los Estados miembros con arreglo a la información que estos mismos le suministran. En algunos ámbitos técnicos, la Comisión procede a comprobar la validez de las denuncias presentadas por los agentes económicos o sociales. En otras áreas, la comprobación de la conformidad de las medidas nacionales se delega en expertos acreditados. En función de la relevancia de los problemas detectados, también se elaboran informes relativos a la transposición e implementación de directivas (hormonas, inseminación artificial, tasas veterinarias, transporte de ganado, etc.). Por último, en ciertos casos, la normativa comunitaria ha instituido comités de seguimiento del proceso de transposición de las directivas, por ejemplo, en materia de IVA o de derechos de importación. Sin embargo, ello no significa que la Comisión esté en condiciones de garantizar a cada Estado miembro o a cada empresa que los servicios o productos procedentes de los demás Estados cumplen con los requisitos de la normativa comunitaria, lo cual pone en entredicho la eficacia real del reconocimiento mutuo.

Los informes anuales de la Comisión sobre la aplicación del Derecho comunitario reflejan en cierta medida el grado de fidelidad de cada Estado con respecto a los compromisos comunitarios. También constituyen indicadores interesantes del nivel de eficiencia de las distintas administraciones nacionales. Como ha puesto de manifiesto el Tribunal de Cuentas de la Comunidad (TC, 1994), los problemas de implementación obedecen a menudo a la complejidad excesiva de la normativa comunitaria y a la lentitud de los procedimientos administrativos. Sin embargo, más generalmente, los motivos de incumplimiento son imputables a otro tipo de factores: la escasa información de los ciudadanos, la falta de capacidad burocrática y las resistencias procedentes de la propia administración o de los sectores económicos. La aplicación en España de la política medioambiental comunitaria es claramente ilustrativa a este respecto (MORATA y Font, 1996).

\section{Los nuevos retos administrativos.}

La puesta en marcha efectiva del Mercado Unico constituye, sin lugar a dudas, una de las principales tareas que aguardan a la administración comunitaria en los próximos años. El Libro Blanco relativo a la realización efectiva del mercado interno (Commission, 1985) preveía la adopción de doscientas ochenta medidas antes de 1993. La mayoría de estas disposiciones han sido adoptadas en forma de Directivas que, por definición, exigen algun tipo de actuación normativa por parte de los Estados miembros, lo cual significa millares de actos legales y administrativos que implican tanto a los ministerios, como a las instituciones regionales y locales, y a un sinfín de entes públicos y semipúblicos con la finalidad de conseguir estándares similares en el conjunto de la Comunidad. Además, el principio del reconocimiento mutuo de las regulaciones y estándares nacionales, un sutil mecanismo de reducción del poder de armonización de la Comisión con la excusa de la desregulación, tiende a aumentar la competencia entre las distintas administraciones nacionales, ya que, para conseguir los mejores resultados posibles, cada Estado debe anticipar los niveles más eficientes. Sin embargo, el reconociento mutuo depende demasiado de la voluntad de los actores nacionales para ser algo más que una solución de emergencia ante los previsibles fallos del mercado europeo (Majone, 1992).

Por otra parte, cuando existen necesidades superiores, como la protección de la salud, la seguridad, el medio ambiente o los derechos de los consumidores, y persisten las diferencias entre las legislaciones nacionales, el reconocimiento mutuo resulta inviable (SUTHERLAND, 1992b). Paralelamente, la Comisión está lanzando nuevas iniciativas tendentes a desarrollar el mercado interno (Comisión, 1993b). Por lo tanto, la regulación seguirá siendo necesaria en el seno de la Comunidad para afrontar los problemas derivados de la puesta en marcha del Mercado Unico. Mediante dos ejemplos - la gestión de las aduanas europeas y la regulación del riesgo- trataremos de demostrar que la administración de los nuevos problemas que se plantean no constituye una cuestión de mera rutina, sino de mejora efectiva de la capacidad de gestión.

Desde el punto de vista de la opinión pública, la supresión de las fronteras físicas entre los Estados miembros simboliza mejor que nada el significado del Mercado Unico. La necesidad 
de proceder a su progresiva eliminación se basaba en los costes económicos generados y en los inconvenientes de todo tipo que las barreras internas representan para el público en general. Sin embargo, la decisión correspondiente no es sino la primera fase de un proceso largo y complejo. En primer lugar, el mantenimiento de las barreras externas de la CE comporta la integración de los servicios aduaneros de los distintos Estados, lo cual plantea a su vez problemas de organización y gestión a escala comunitaria. En 1991, un estudio relativo a "la gestión de las aduanas europeas" (KEARNY, 1991) concluía que "el funcionamiento actual de la Unión Aduanera todavía no ha alcanzado el grado de uniformidad y de eficiencia que exige la realización del Mercado Interno". La integración de los servicios aduaneros se ve afectada de forma simultánea por tres tipos de procesos (SUTHERLAND, 1992a). El primero se refiere al desmantelamiento de los controles internos transfronterizos y a las organizaciones implicadas. El segundo a la redefinición de las funciones administrativas: los controles fronterizos incluyen distintas funciones relativas a inmigración y fiscalidad, así como a tráfico de drogas, terrorismo, salud animal y bienestar, incluyendo la protección de las especies en peligro y la prevención de las enfermedades. El tercer cambio se refiere al diseño y desarrollo del nuevo sistema de control de las fronteras externas. A esta problemática se añade el impacto social y territorial de la Unión Aduanera. Antes de la puesta en marcha del proceso, los servicios aduaneros estatales empleaban alrededor de ciento treinta mil personas, repartidas entre unos dos mil puestos en el conjunto de la Comunidad de los Doce.

La gestión de la política de abolición de fronteras requiere, por consiguiente, una coordinación a tres niveles: interno (dentro de las administraciones nacionales), horizontal (entre éstas) y vertical (entre éstas y la Comisión). Sin embargo, el sistema inicial se caracterizaba por la existencia de doce administraciones separadas con grados de desarrollo y patrones de actuación distintos. Por ello, no es de extrañar que una de las conclusiones más destacadas del citado estudio consistiera en la detección de "un déficit de gestión en la administración aduanera al nivel europeo" y en "la necesidad de definir un mecanismo susceptible de introducir criterios de gestión comunes de cara a la puesta en práctica de la Unión Aduanera" (KEARNEY, 1991). El problema reside en la ausencia de los conocimientos necesarios para planificar y gestionar esta transformación institucional. En particular, el estilo tradicional de la Comisión no concuerda excesivamente con las exigencias de una gestión no jerárquica que dicho cambio plantea (MetCalfE, 1992).

El segundo ejemplo se refiere a la regulación del riesgo en el ambiente competitivo generado por el Mercado Interno (Dehousse et al., 1992). El concepto de "riesgo" es muy amplio, ya que incluye todo tipo de actividades relacionadas con la salud, la seguridad y el medio ambiente: maquinaria peligrosa, manipulación de sustancias peligrosas, alimentos, medicamen- tos, contaminación, juguetes, electrodomésticos, ingeniería genética, etc. De algún modo, el riesgo es algo consustancial a las actividades humanas y, en especial, al desarrollo tecnológico. las empresas asumen riesgos de forma deliberada como consecuencia de la competitividad y de la búsqueda de beneficios. Cualquier intento de regulación sistemática del riesgo en el ámbito del Mercado Unico resultaría contradictorio con la lógica actual del proceso económico. Por tanto, el problema consiste en hallar un compromiso entre la regulación del riesgo y el mercado. En este sentido, resulta necesario garantizar la uniformidad o equivalencia de los niveles de seguridad de los productos que circulan en el mercado comunitario. Sin embargo, sería excesivo -además de escasamente viable- imponer tecnologías avanzadas a todos y cada uno de los productos y regiones de la Comunidad (Dehousse et al., 1992).

Si bien la Comunidad ha tratado de regular, a la vez, las distintas formas de riesgo y los límites entre los riesgos socialmente aceptables e inaceptables, las estructuras legislativas y judiciales tradicionales no bastan para conseguir tales objetivos. Al mismo tiempo, la supervisión del proceso de transposición de las normas comunitarias en normas nacionales no garantiza por sí sola una regulación eficaz. Por ahora, las mayores responsabilidades corresponden a la Comisión y a la red de comités consultivos, reguladores y de gestión completados por acuerdos de cooperación con los organismos europeos de estandarización en materia de armonización técnica y fijación de estándares. Sin embargo, las deficiencias de la comitología constituyen un grave inconveniente a la hora de afrontar una problemática que supera las posibilidades del sistema de regulación comunitario (DeHousse et al., 1992).

La especificidad de la regulación del riesgo implica la necesidad de reaccionar ante situaciones imprevisibles, desarrollando nuevos estándares a medida que aparecen nuevos problemas. La operatividad de dicho sistema requiere una comunicación eficiente entre las distintas zonas del territorio comunitario y la Comisión al objeto de alertar a los evaluadores descentralizados. Sin embargo, la Comunidad no dispone en la actualidad de los recursos técnicos y administrativos necesarios para establecer un sistema de estas características. La gestión del riesgo exigiría el establecimiento de una red de reguladores nacionales, expertos y ONGs capaz de intervenir antes y después de las decisiones. El estudio concluye que: "la coordinación y supervisión comunitarias deberían reforzarse mediante la creación de agencias especializadas que se beneficiarian de los conocimientos disponibles en los distintos Estados miembros. Las funciones de dichos organismos deberían limitarse a la asistencia y, si fuera necesario, al reforzamiento de las políticas reguladoras seguidas en cada Estado" (DeHousse et al., 1992:77). 


\section{La respuesta de la Comisión: la cooperación administrativa}

La respuesta de la Comisión a esta problemática ha consistido en el establecimiento de un programa estratégico con objeto de conseguir una mayor transparencia en su actuación implicando al conjunto de los actores públicos, económicos y sociales afectados (ComISIÓN, 1993a). Dicha estrategia tiene una doble dimensión: conseguir una mayor cooperación entre la Comisión y los Estados miembros en la aplicación y la gestión efectiva de las medidas previstas en el Libro Blanco y movilizar a los operadores económicos para el desarrollo del mercado interno.

El informe Sutherland (1992b), relativo al funcionamiento del Mercado Interno después de 1992, hacía hincapié en el principio de cooperación interadministrativa como elemento esencial para el funcionamiento del mercado interior. En su respuesta a dicho informe, la Comisión afirmaba que la puesta en práctica de las recomendaciones contenidas en el informe "reforzaría los efectos económicos del mercado interior, así como su dimensión política" (Comisión, 1993). Con arreglo a ello, el programa estratégico Aprovechar al Máximo el Mercado Interior, presentado bajo forma de Comunicación de la Comisión al Consejo (Comisión, 1993b) incluye un apartado dedicado a la "Gestión del Mercado Interno" en el que se delimitan tanto el marco general como las medidas de apoyo a la cooperación administrativa. Estas prevén las medidas siguientes:

a) el desarrollo de una red de comunicación e intercambio de datos para las administraciones de la Unión;

b) la elaboración de guías de interpretación de la legislación y la publicación de comunicaciones interpretativas por parte de la Comisión;

c) la continuación de los programas existentes de intercambio de funcionarios entre Estados miembros (KAROLUS, de septiembre de 1992, relativo al intercambio de funcionarios dedicados a la puesta en prática del mercado interior; y MATTHAEUS, de 1991, para la formación de funcionarios de aduanas, completado en octubre de 1993 mediante METTHAEUS-impuestos, dedicado a funcionarios de la administración tributaria);

d) la organización de reuniones, seminarios y conferencias ad boc sobre determinados aspectos de la aplicación del derecho comunitario;

e) la recogida, traducción y transmisión de información sobre estructuras y prácticas de aplicación de los distintos Estados miembros;

f) la evaluación de la posible necesidad de ayuda comunitaria para el reforzamiento de las estructuras administrativas.
Conforme al compromiso adquirido en dicho programa, la Comisión presentó en febrero de 1994 una Comunicación al Consejo y al Parlamento "relativa al desarrollo de la cooperación administrativa para la aplicación y el cumplimiento de la legislación comunitaria en el mercado interior" (COMSIÓN, 1994). La Comunicación analiza el estado de la cooperación administrativa en el ámbito del mercado interior y propone de medidas para garantizar su eficacia y ulterior desarrollo. El marco propuesto para la cooperación administrativa se basa en los siguientes elementos:

- la obligación de asistencia mutua entre las administraciones implicadas, por ejemplo, mediante el intercambio de información sobre la conformidad de los productos o la notificación de los requisitos de control y de sus modificaciones;

- la obligación de transparencia de los mecanismos nacionales de aplicación y cumplimiento de la normativa comunitaria, para lo cual se sugiere el nombramiento de "corresponsales" en cada sector y en cada Estado miembro cuya misión consistirá en actuar como interlocutores permanentes de la Comisión y de los demás Estados miembros;

- el principio de la proporcionalidad, entendido como criterio de regulación de las relaciones entre las distintas administraciones: "las autoridades han de limitar sus peticiones reciprocas al mínimo imprescindible para resolver el asunto de que se trate".

- el principio de la confidencialidad, es decir, "la aplicación de normas y procedimientos de seguridad comunes y medidas técnicas, como el suministro de mecanismos de protección de la información".

La propuesta de la Comisión contempla igualmente la organización de un "programa de debates" en relación a los sectores en los que la experiencia de cooperación es inexistente, limitada o requiere una reformulación.

Por último, con respecto a la aplicación del principio del reconocimiento mutuo, tras analizar los obstáculos que impiden todavía la plena aplicación de dicho principio, la Comisión (1993c) ha propuesto el establecimiento de un procedimiento de información recíproca desde el momento mismo en que un Estado miembro invoca alguna de las excepciones legalmente previstas. El nuevo procedimiento, aplicable a los casos no previstos en los procedimientos de notificación ya vigentes, presenta la ventaja de garantizar una mayor seguridad jurídica a los operadores económicos y a los consumidores.

\section{Conclusiones}

En los últimos años, la Comisión Europea ha sido objeto de duras críticas basadas en el exceso de reglamentismo y en las prácticas burocráticas. Sin embargo, la Comisión no actúa por su cuenta y riesgo, sino que su margen de decisión depende de la voluntad de acuerdo de las administraciones nacionales 
manifestada, no sólo por los representantes permanentes en Bruselas, sino también por un verdadero ejército de comités de todo tipo compuestos por funcionarios, expertos y representantes de los grupos de interés nacionales y transnacionales. Los comportamientos burocráticos, el exceso de tecnocracia y la falta de transparencia de la decisiones resultan de la complejidad de las políticas y, ante todo, de las reglas decisorias y de los controles impuestos por los propios gobiernos. Por tanto, las críticas a la Comisión esconden en realidad las peculiaridades institucionales del sistema comunitario, tanto en lo relativo a los mecanismos de control de las decisiones como a su legitimidad democrática.

Los ejemplos analizados ponen de relieve la dimensión de los cambios organizativos y de gestión que deberán afrontar en los próximos años el conjunto de las administraciones responsables de la implementación de las políticas comunitarias. Este reto colectivo implica un replanteamiento de los métodos de gestión tradicionales, esencialmente basados en la regulación. No parece descabellado hipotizar que la Comisión ha podido desarrollar su imponente aparato regulador central gracias a la desconfianza de cada uno de los Estados miembros con respecto a la voluntad reguladora de los demás. Sin embargo, la regulación no basta por sí sola para garantizar el funcionamiento del sistema comunitario puesto que no existen los medios necesarios para conseguir una aplicación homogénea de la legislación europea en todos los Estados miembros. Dicha situación es especialmente preocupante habida cuenta, sobre todo, de los peligros derivados del principio del reconocimiento mutuo. Al objeto de paliar dicho déficit, la Comisión ha elaborado recientemente un programa específico tendente a fomentar la cooperación entre el conjunto de las administraciones implicadas en la aplicación y el cumplimiento de la legislación comunitaria en el Mercado Interior.

En cualquier caso, está claro que las iniciativas de la Comisión no bastan por sí solas para garantizar el funcionamiento equilibrado del Mercado Interno. Las distintas administraciones nacionales (estatales, regionales y locales) deben integrar el elemento europeo en sus culturas organizativas en un contexto que se caracterizará, cada vez más, por la exigencia de cooperación y también de una mayor competitividad, resultado inevitable del principio del reconocimiento mutuo. La aplicación del principio de subsidiariedad debería conducir a reforzar las funciones ejecutivas y de control de las administraciones nacionales, dejando a la Comisión las tareas relativas a la definición de los estándares técnicos, la coordinación general y la evaluación de las políticas, incluyendo la creación de organismos independientes, según el modelo de las agencias federales estadounidenses, capaces de asesorar a los implementadores nacionales. En dicho contexto, la información constituye un recurso fundamental para la aplicación correcta de las políticas comunitarias (inspecciones técnicas, contratos de obras públicas, etc.). El acceso de los ciudadanos y los grupos a dicha información es un requisito indispensable para la transparencia democrática y, en definitiva, para la afirmación misma de la Unión Europea.

- Departamento de Ciencia Política y Derecho Público. Universidad Autónoma de Barcelona

\section{Bibliografia}

BlumanN, C., "La Comitologie: L'exercice de la fonction exécutive dans la Communauté Européennen, en Ch. Engel \& W. Wessels (eds.), From Luxembourg to Masstricht, Bonn: Institut für Europäische Politik, 1992.

CASSESE, S. (ed.), The European Administration, Bruselas: ILAS, 1988.

CiavariNI AzZI, G. (ed.), L'application du droit communautaire dans les Etats membres: une vue d'ensemble, Maastricht: Institut Européen d'Administration Publique, 1986.

COMISIÓN dE las COMUNIDAdES EuROPEAS (1993), "Un diálogo abierto y estructurado entre la Comisión y los grupos de interés", Comunicación de la Comisión al Consejo y al Parlamento Europeo, COM(93/C 63/02).

Comisión (1993a), Comunicación de la Comisión relativa al tratamiento de los casos de urgencia en el contexto de la aplicación de las normas comunitariasSeguimiento del Informe Sutberland, COM(93) 430 final.

COMISIÓN (1993b), "Aprovechar al máximo el mercado interior: Programa Estratégico", СOM(93) 632 final.
COMISIÓN (1993c), "Gestión del reconocimiento recíproco de las normas nacionales después de 1992", Comunicación de la Comisión al Consejo y al Parlamento Europeo, COM(93) 669 final.

Comision (1994), Comunicación de la Comisión al Consejo y al Parlamento Europeo relativa al desarrollo de la cooperación administrativa para la aplicación y el cumplimiento de la legislación comunitaria en el mercado interior, COM(94) 29 final.

COMmISSION OF THE EUROPEAN COMMUNITES, Completing the Internal Market, Luxemburgo: Oficina de Publicaciones Oficiales de las Comunidades Europeas, 1985.

Commission of the European Communtmes (1993), "Working Document of the Commission on a Strategic Programme on the Internal Market", Communication from the Commission to the Council and the European Parliament, COM(03) 256 final.

DeHousSE, R., y Majone, G., "The Institutional Dymamics of European Integration: Towards a New Paradigm", Ponencia presentada en la European Commu- 


\section{Bibliografia}

nity Studies Association, Third Biennal International Conference, Washington, D.C., 27-29 de mayo 1993.

DeHousse et al. (1993), "Europe After 1992: New Regulatory Strategies", EUI Working Papers $L A W$, núm. 92/31, Instituto Universitario Europeo, Florencia.

DEwost, J. L., "Intérêts Nationaux et Intérêt Communautaire dans les Décisions de la Commission et du Conseil", Revue française d'administration publique, núm. 63, julio-septiembre 1992, pp. 371-381.

EuRocitites, The European Association of Metropolitan Cities, Bruselas, marzo 1994.

GonzÁlEz, E., Manual del Negociador en la Comunidad Europea, Madrid: Oficina de Información Diplomática, 1992.

HaY, R., "La Comisión Europea y la Administración de la Comunidad", Comisión de la Comunidad Europea, Luxemburgo: Oficina de Publicaciones Oficiales de la Comunidad Europea, 1989.

KaSSIM, H., y WRIGHT, V., "The Role of National Administrations in the DecisionMaking Processes of the European Community", Rivista Trimestrale di Diritto Pubblico, 1992, pp. 833-850.

KfarNy, D., "The Management of European Costums", Administration, 39 (3), 1991.

LODGE, J., "EC policymaking: Institutional dynamics", en J. Lodge (ed.), The European Community and the Challenge of the Future, Londres: Pinter Publishers, 1993.

Ludiow, P., "The European Commission", en Keohane \& Hoffmann (eds.), The New European Community: Decision Making and Institutional Change, Westview: Boulder, Col., 1992.

MajONE, G., "Regulatory Federalism in the European Community", Environment and Planning C: Government and Policy, 1992, vol. 10, pp. 299-316.

Majone, G., "L'innovazione politica nella Comunità Europea", Stato e Mercato, núm. 37, abril 1993, pp. 153-162.

MAZEY, S., y RichaRDSON, J., "Pressure groups and lobbying in the EC", en J. Lodge (ed.), The European Community and the Challenge of the Future, 2. ${ }^{2}$ edición, Londres: Pinter, 1993.

MetCalfE, L., "Après 1992: La Commission Pourra-t-elle gérer l'Europe?", Revue française d'administration publique, núm. 63, julio-septiembre 1992, pp. 401412.

Meny, Y., y Thoenig, J. C., Las Politicas Püblicas, Barcelona: Ariel, 1992.

Molins, J. M., y Morata, F., "Spain: Rapid Arrival of Latecomer", en R. Van Schendelen (ed.), National Public and Private EC Lobbying, op. cit.

Morata, F., "El Estado de las Autonomías y las Estrategias Regionales en la Europa de los 90", en M. Arenilla et al., La Europa de las Regiones: Una perspectiva intergubernamental, Universidad de Granada, 1994.

Morata, F., y Font, N., "Environmental Policy and Public Administration in Spain: A Marriage of Convenience officiated by the EC, en K. Hanf y A. I. Jen- sen (eds.), Public Administration and Environment in Europe (próxima publicación), 1995.

NuGeNT, The Government and Politics of the European Community, 2nd. ed., Londres: Macmillan and Durham: Duke University Press, 1991.

PAG, S., "The Relations between the Commission and National Bureaucracies", en S. Cassese (ed.), The European Administration, op. cit.

PETERS, G., "Bureaucratic Politics and the Institutions of the European Community", en A. M. Sbragia (ed.), Euro-Politics, Washington, D.C.: The Brookings Institution, 1992.

SCHmrTter, PH., "The European Community as an Emergent and Novel form of Political Domination", Centro de Estudios Sociales Avanzados, Estudio/Working Paper 1991/26, september 1991.

SIEDENTOPF, H., y ZILIFR, J. (eds.), L'Europe des Administrations, 2 vols., Bruselas: Bruylant, 1988.

SPIERENBURG RePort, "Proposals For Reform of The Commission of the European Communities and its Services", Report made at the request of the Commission by an Independent Review Body under the chaimanship of Mr Dirk Spierenburg, Bruselas, 24 de septiembre de 1979.

Sutherland, P. (1992a), "Progress to European Union - A Challenge for the Public Service", EIPASCOPE, Institut Européen d'Administration Publique, núm. 1992/3, pp. $1-5$.

Sutherland, P. (1992b), "Le Marché Intérieur Après 1992: Répondre au Défi", Rapport présenté à la Commission par le Groupe à haut niveau sur le fonctionnement du marché intérieur, Bruselas, IIL/21/1992.

TC (TRIBUNAL DE CUENTAS), Sintesis del Informe Anual del Tribunal de Cuentas de las Comunidades Europeas sobre el Ejercicio 1992, Comunidades Europeas, Luxemburgo, 1993.

TOONEn, T. A. J., "Europe of the Administrations: The Challenges of ' 92 (and Beyond), Public Administration Review, núm. 2, marzo/abril 1992, pp. 108-115.

VAN SCHENDElEN, R., "Introduction: The relevance of National Public and Private EC Lobbying", en R. Van Schendelen (ed.), National Public and Private EC Lobbying, Aldershot: Dartmouth, 1993.

WAUACE, H. et al. (eds.), Policy-Making in the European Community, Chichester: J. Wiley \& Sons.

WALACE, W., "Less than a Federation, More than a Regime: The Community as a Political System", en H. WELACE et al., Policy-Making in the European Community, Chichester: John Wiley \& Sons, 1983.

WeILER, J., Il Sistema Comunitario, Bolonia: Il Mulino, 1984.

WESSELS, W., "Administrative Interaction", en W. Wallace (ed.), The Dymamics of European Integration, Londres: Pinter Publishers, 1990.

WESSELS, W., "The EC Council: The Community's Decisionmaking Center", en Keohane y Hoffmann (eds.), The New European Community: Decision-Making and Institutional Change, op. cit. 